\title{
On Different Type Solutions of Boundary Value Problems*
}

\section{Maria Dobkevich $^{a}$ and Felix Sadyrbaev ${ }^{a}$}

\author{
${ }^{a}$ Institute of Mathematics and Computer Science, University of Latvia \\ Raynis boulevard 29, Riga, Latvia \\ E-mail(corresp.): felix@latnet.lv
}

Received June 15, 2015; revised June 14, 2016; published online September 15, 2016

\begin{abstract}
We consider boundary value problems of the type $x^{\prime \prime}=f\left(t, x, x^{\prime}\right), \quad(*)$ $x(a)=A, x(b)=B$. A solution $\xi(t)$ of the above BVP is said to be of type $i$ if a solution $y(t)$ of the respective equation of variations $y^{\prime \prime}=f_{x}\left(t, \xi(t), \xi^{\prime}(t)\right) y+$ $f_{x^{\prime}}\left(t, \xi(t), \xi^{\prime}(t)\right) y^{\prime}, y(a)=0, y^{\prime}(a)=1$, has exactly $i$ zeros in the interval $(a, b)$ and $y(b) \neq 0$. Suppose there exist two solutions $x_{1}(t)$ and $x_{2}(t)$ of the BVP. We study properties of the set $S$ of all solutions $x(t)$ of the equation $(*)$ such that $x(a)=A$, $x_{1}^{\prime}(a) \leq x^{\prime}(a) \leq x_{2}^{\prime}(a)$ provided that solutions extend to the interval $[a, b]$.
\end{abstract}

Keywords: boundary value problem, multiple solutions, existence.

AMS Subject Classification: 34B15.

\section{Introduction}

The classical object in the theory of nonlinear boundary value problems [1], $[4],[3]$ is the below problem

$$
\begin{aligned}
& x^{\prime \prime}=f\left(t, x, x^{\prime}\right), \\
& x(a)=A, \quad x(b)=B .
\end{aligned}
$$

The main issues considering the qualitative analysis of this type problems are the existence of a solution, conditions for the uniqueness, estimates of the number of solutions, properties of solutions. We believe that the main information concerning properties of solutions and multiplicity of solutions can be treated using the notion of the type of a solution. The type of a solution for the problem (1.1), (1.2) can be introduced as in [7].

If the function $f$ in (1.1) is continuously differentiable and equations of variations

$$
y^{\prime \prime}=f_{x}\left(t, \xi(t), \xi^{\prime}(t)\right) y+f_{x^{\prime}}\left(t, \xi(t), \xi^{\prime}(t)\right) y^{\prime}
$$

* This research is supported by the European Social Fund within the project Nr. 2013/0024/1DP/1.1.1.2.0/13/APIA/VIAA/045. 
can be considered, the definition of the type of a solution can be made in a simplified form.

We say that a solution $\xi(t)$ of the BVP (1.1), (1.2) is of type $i$, where $i=0,1, \ldots$, if a solution $y(t)$ of the respective equation of variations (1.3) given with the initial conditions

$$
y(a)=0, \quad y^{\prime}(a)=1
$$

has exactly $i$ zeros in the interval $(a, b)$ and $y(b) \neq 0$.

For instance, the trivial solution of the BVP $x^{\prime \prime}=-x^{3}, x(0)=0, x(b)=0$ is of type zero for any $b>0$ since a solution $y(t)$ of the Cauchy problem $y^{\prime \prime}=0$, $y(0)=0, y^{\prime}(0)=1$ has no zeros for $t>0$.

We assume that there exist two solutions $u(t)$ and $v(t)$ of the BVP (1.1), (1.2) and that all intermediate solutions (that is, solutions with the initial data $\left.x(a)=A, u^{\prime}(a) \leq x^{\prime}(a) \leq v^{\prime}(a)\right)$ are extendable to the interval $[a, b]$. These intermediate solutions constitute a set $S(u, v)$. We study properties of this set which relate to location of solutions, their interrelation and the number of solutions of BVP (1.1), (1.2).

The paper is organized as follows. In Section 2 we give some preliminary facts on which to base our results. In Section 3 the specific diagram is considered that makes it easier to formulate the results. In Section 4 sets of solutions of the equation are treated which are associated with two given solutions of BVP.

\section{Preliminaries}

Denote $x(t, \gamma)$ a solution of the Cauchy problem

$$
x^{\prime \prime}=f\left(t, x, x^{\prime}\right), \quad x(a)=A, \quad x^{\prime}(a)=\gamma .
$$

Suppose there are two solutions $u:=x\left(t, \gamma_{*}\right)$ and $v:=x\left(t, \gamma^{*}\right)$ of the BVP (1.1), (1.2), that is, $u(b)=v(b)=B$. Let, for definiteness, $u^{\prime}(a)=\gamma_{*}<v^{\prime}(a)=\gamma^{*}$. The equality sign is not possible due to the uniqueness of solutions of the initial value problems.

We consider a set $S(u, v)$ ( $S$ in short) of all solutions of the Cauchy problem (2.1), where $\gamma \in\left[\gamma_{*}, \gamma^{*}\right]$. Suppose the following condition is fulfilled.

(C) all elements of $S$ extend to the interval $[a, b]$.

Lemma 1. There exists a constant $M>0$ such that $|x(t)|<M$ and $\left|x^{\prime}(t)\right|<$ $M \forall t \in[a, b]$ for any $x \in S$.

Follows from Theorem 15.1 in [5]. This theorem when interpreted for the case under consideration says that the set $S$ is compact and connected in $C^{1}[a, b]$. Therefore both sets $x(t)$ and $x^{\prime}(t)$ are equibounded.

Lemma 2. There exists a number $\delta(M)>0$ such that for any two different elements $x_{1}$ and $x_{2}$ of $S$ the following implication holds:

$\left\{x_{1}\left(t_{1}\right)=x_{2}\left(t_{1}\right), x_{1}\left(t_{2}\right)=x_{2}\left(t_{2}\right), t_{1}, t_{2} \in[a, b], t_{1} \neq t_{2}\right\} \Rightarrow\left|t_{1}-t_{2}\right|>\delta(M)$. 
Proof. Let $x_{1}$ and $x_{2}$ be different elements of $S$. Then the difference $z(t)=$ $x_{2}(t)-x_{1}(t)$ satisfies

$$
\begin{aligned}
& z^{\prime \prime}(t)=x_{2}^{\prime \prime}(t)-x_{1}^{\prime \prime}(t)=f\left(t, x_{2}(t), x_{2}^{\prime}(t)\right)-f\left(t, x_{1}(t), x_{1}^{\prime}(t)\right) \\
& =f\left(t, x_{2}(t), x_{2}^{\prime}(t)\right)-f\left(t, x_{1}(t), x_{2}^{\prime}(t)\right)+f\left(t, x_{1}(t), x_{2}^{\prime}(t)\right)-f\left(t, x_{1}(t), x_{1}^{\prime}(t)\right) \\
& =\frac{f\left(t, x_{2}(t), x_{2}^{\prime}(t)\right)-f\left(t, x_{1}(t), x_{2}^{\prime}(t)\right)}{x_{2}(t)-x_{1}(t)} z(t) \\
& \quad+\frac{f\left(t, x_{1}(t), x_{2}^{\prime}(t)\right)-f\left(t, x_{1}(t), x_{1}^{\prime}(t)\right)}{x_{2}^{\prime}(t)-x_{1}^{\prime}(t)} z^{\prime}(t)=\varphi(t) z+\psi(t) z^{\prime},
\end{aligned}
$$

where

$$
\begin{gathered}
\varphi(t)= \begin{cases}\frac{f\left(t, x_{2}(t), x_{2}^{\prime}(t)\right)-f\left(t, x_{1}(t), x_{2}^{\prime}(t)\right)}{x_{2}(t)-x_{1}(t)}, & x_{2}(t) \neq x_{1}(t), \\
f_{x}\left(t, x_{1}(t), x_{2}^{\prime}(t)\right), & x_{2}(t)=x_{1}(t) ;\end{cases} \\
\psi(t)= \begin{cases}\frac{f\left(t, x_{1}(t), x_{2}^{\prime}(t)\right)-f\left(t, x_{1}(t), x_{1}^{\prime}(t)\right)}{x_{2}^{\prime}(t)-x_{1}^{\prime}(t)}, & x_{2}(t) \neq x_{1}(t), \\
f_{x^{\prime}}\left(t, x_{1}(t), x_{1}^{\prime}(t)\right), & x_{2}(t)=x_{1}(t)\end{cases}
\end{gathered}
$$

The functions $\varphi(t)$ and $\psi(t)$ by construction are continuous in the interval $[a, b]$. The following estimates hold for $t \in[a, b]$ :

$$
\begin{aligned}
& |\varphi(t)| \leq M_{1}(M):=\max \left\{\left|f_{x}(t, x, y)\right|: a \leq t \leq b,|x| \leq M,|y| \leq M\right\} \\
& |\psi(t)| \leq M_{2}(M):=\max \left\{\left|f_{y}(t, x, y)\right|: a \leq t \leq b,|x| \leq M,|y| \leq M\right\}
\end{aligned}
$$

Then by the Vallée Poussin Theorem [6, p. 122] the difference between any two zeros of any solution of the equation $z^{\prime \prime}=\varphi(t) z+\psi(t) z^{\prime}$ is not less than the constant

$$
\delta(M)=\left(\sqrt{9 M_{2}^{2}+24 M_{1}^{2}}-3 M_{2}\right) / 2 M_{1} .
$$

Since $M_{1}$ and $M_{2}$ depend only on $M$, the proof is completed.

\section{Triangle region}

Consider the triangle region depicted in Figure 1.

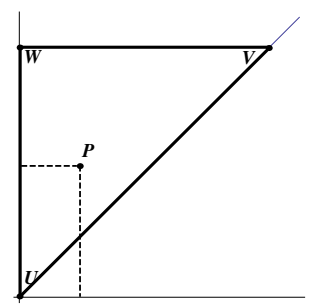

Figure 1. The triangle region: $U=\left(\gamma_{*}, \gamma_{*}\right), W=\left(\gamma_{*}, \gamma^{*}\right), V=\left(\gamma^{*}, \gamma^{*}\right), P=\left(\gamma_{1}, \gamma_{2}\right)$. 
This region is associated with the set $S$ and solutions $u=x\left(t, \gamma_{*}\right), v=$ $x\left(t, \gamma^{*}\right)$. There are $x^{\prime}(a)$ on both axis. The vertices are $U=\left\{\gamma_{*}, \gamma_{*}\right\}, V=$ $\left\{\gamma^{*}, \gamma^{*}\right\}, W=\left\{\gamma_{*}, \gamma^{*}\right\}$. Any point $P$ with the coordinates $\left(\gamma_{1}, \gamma_{2}\right)$ in this region corresponds to a pair of solutions $x\left(t, \gamma_{1}\right)$ and $x\left(t, \gamma_{2}\right)$. The vertex $W$ corresponds to the pair $x\left(t, \gamma_{*}\right)$ and $x\left(t, \gamma^{*}\right)$. Any point on the side $U W$ corresponds to $x\left(t, \gamma_{*}\right)$ and $x(t, \gamma)$, where $\gamma_{*}<\gamma \leq \gamma^{*}$. Consequently any point on the segment $W V$ relates to an ordered couple of solutions $x(t, \gamma), x\left(t, \gamma^{*}\right)$. Any point on the hypotenuse $U V$ is associated with $x(t, \gamma), x(t, \gamma)$, where $\gamma_{*} \leq \gamma \leq \gamma^{*}$. We collect some useful information at any point $P$ of this region included vertices and the sides. For any $i=1,2, \ldots$ we define functions $Z_{i}\left(\gamma_{1}, \gamma_{2}\right)$ which are the $i$-th zero (if any) of the difference $x\left(t, \gamma_{1}\right)-x\left(t, \gamma_{2}\right)$ in the interval $(a, b]$.

Of course, $Z_{1}<Z_{2}<\ldots$. Especially interesting is the case of $Z_{i}\left(\gamma_{1}, \gamma_{2}\right)=b$. This means that graphs of solutions $x\left(t, \gamma_{1}\right)$ and $x\left(t, \gamma_{2}\right)$ intersect at $t=b$ (they may intersect at a point $(b, \cdot)$ other than $(b, B)$ and therefore need not to be solutions of the BVP) and this point of intersection is $i$-th, that is, there are $i-1$ cross points of the graphs of both solutions in the interval $(a, b)$.

Points on the side $U W$ correspond to couples of solutions $x\left(t, \gamma_{*}\right)$ and $x(t, \gamma)$ and the respective values of $Z_{i}\left(\gamma_{*}, \gamma\right)$ reflect intersections of graphs of the respective solutions. Similarly for the side $W V$. Points on the hypotenuse $U V$ are associated with the couples $(x(t, \gamma), x(t, \gamma))$. The functions $Z_{i}(\gamma, \gamma)$ for these particular couples of solutions are defined as $i$-th zeros of a solution $y(t)$ of equation of variations

$$
y^{\prime \prime}=f_{x}\left(t, x(t, \gamma), x^{\prime}(t, \gamma)\right) y+f_{x^{\prime}}\left(t, x(t, \gamma), x^{\prime}(t, \gamma)\right) y^{\prime}
$$

considered with the initial conditions $y(a)=0, y^{\prime}(a)=1$. This is justified by the fact that $y(t)$ is an approximation of the difference $x\left(t, \gamma_{1}\right)-x(t, \gamma)$ as $\gamma_{1} \rightarrow \gamma$, where $x\left(t, \gamma_{1}\right)$ and $x(t, \gamma)$ are solutions of equation $x^{\prime \prime}=f\left(t, x, x^{\prime}\right)$ subject to the initial conditions $x\left(a, \gamma_{1}\right)=x(a, \gamma)=0, x^{\prime}\left(a, \gamma_{1}\right)=\gamma_{1}, x^{\prime}(a, \gamma)=\gamma$ respectively. Then functions $Z(\cdot, \cdot)$ remain continuous on the entire triangle $U W V$.

To illustrate the above introduced functions we provide two pictures with traces $Z_{i}(b):=\left\{\right.$ section of the graph of $Z_{i}(\cdot, \cdot)$ by the plane $\left.t=b\right\}$ on the triangle region.

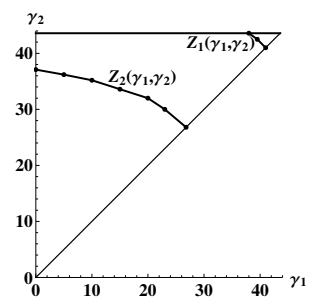

Figure 2. $Z_{1}(1), Z_{2}(1)$ for BVP $x^{\prime \prime}=x^{3}-(5 \pi / 2)^{2} x, x(0)=x(1)=0, u^{\prime}(0)=0$, $v^{\prime}(0)=43.594$ (schematically).

The triangle region for the equation $x^{\prime \prime}=x^{3}-(5 \pi / 2)^{2} x$, considered in the interval $[0,1]$, is depicted in Figure 2. The trivial solution is identified with $u$ 
and $v$ is a solution with the initial conditions $v(0)=0, v^{\prime}(0)=43.594$. The Cauchy problems $x(0)=0, x^{\prime}(0)=\gamma$ were considered, where $\gamma \in[0,43.594]$. For any couple of solutions $x\left(t ; \gamma_{1}\right)$ and $x\left(t ; \gamma_{2}\right)$ the first point of intersection of their graphs is $Z_{1}\left(\gamma_{1}, \gamma_{2}\right)$, the second cross point provides a value for $Z_{2}\left(\gamma_{1}, \gamma_{2}\right)$ and so on. If $Z_{1}\left(\gamma_{1}, \gamma_{2}\right)=1$ for some $\left(\gamma_{1}, \gamma_{2}\right)$ this means that the respective solutions $x\left(t ; \gamma_{1}\right)$ and $x\left(t ; \gamma_{2}\right)$ are equal at $t=1$ for the first time after $t=0$. The segment of a curve $Z_{1}$ in Figure 2 is an evidence of the existence of a continuum of such solutions. It is to be mentioned that these solutions need not to satisfy the boundary condition $x(1)=0$. However the upper point of the segment $Z_{1}$ indicates that there is a solution other than $v$ that like $v$ satisfies the condition $x(1)=0$ and, like $v$, is a solution of the boundary value problem $x(0)=0, x(1)=0$ for the above equation. Similarly, the segment $Z_{2}$ in Fig. 2 points out to $\left(\gamma_{1}, \gamma_{2}\right)$ corresponding to solutions that intersect exactly at $t=1$ for the second time in the interval $(0,1]$. The very left point of the segment $Z_{2}$ corresponds to a solution that, like $u(t) \equiv 0$, satisfies $x(1)=0$ and is a solution of the boundary value problem $x(0)=0, x(1)=0$. The segments $Z_{1}$ and $Z_{2}$ are obtained by joining of points of intersection of the respective solutions calculated by Mathematica. Therefore all values are approximate.

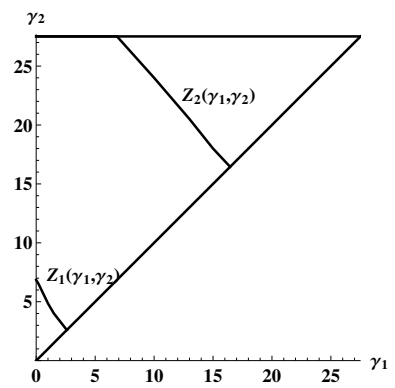

Figure 3. $Z_{1}(1), Z_{2}(1)$ for BVP $x^{\prime \prime}=-2 x^{3}, x(0)=x(1)=0, u^{\prime}(0)=0, v^{\prime}(0)=27.514$ (precisely, since solutions are known analytically).

In Figure 3 the equation $x^{\prime \prime}=-2 x^{3}$ is considered in the interval $[0,1]$. The two base solutions $u$ and $v$ are respectively the trivial one and the solution of the Cauchy problem $v(0)=0, v^{\prime}(0)=27.514$. Since a solution of the Cauchy problem $x(0)=0, x^{\prime}(0)=\gamma$ is known analytically, that is, $x(t ; \gamma)=\sqrt{\gamma} \operatorname{sl}(\sqrt{\gamma} t)$, where $\operatorname{sl} t$ is the lemniscate sine function (computable by Mathematica) both curves $Z_{1}(1)$ and $Z_{2}(1)$ can be constructed and visualized as $Z_{1}(1)=\left\{\left(\gamma_{1}, \gamma_{2}\right) \in(0,27.514) \times(0,27.514), \gamma_{1}>\gamma_{2}:\right.$ the difference $x\left(t, \gamma_{1}\right)-$ $-x\left(t, \gamma_{2}\right)$ has the first zero at $\left.t=1\right\}$. The curve $Z_{2}$ is constructed similarly ("the first zero" should be replaced by "the second zero").

We state a number of simple assertions below which can be proved merely by observing Figure 1.

Proposition 1. If $Z_{i}\left(\gamma_{*}, \gamma\right)=b$ for some $i \in \mathbb{N}$ and $\gamma \in\left(\gamma_{*}, \gamma^{*}\right]$ then $x(t, \gamma)$ is a solution of the $B V P(1.1),(1.2)$ and $x(t, \gamma)$ has $i-1$ points of intersection with $x\left(t, \gamma_{*}\right)$ in the interval $(a, b)$. 
Proof. Since $x(b, \gamma)=x\left(b, \gamma_{*}\right)$ and $x\left(b, \gamma_{*}\right)=B$ it follows that $x(b, \gamma)=B$ also and therefore $x(t, \gamma)$ is a solution of the BVP (1.1), (1.2). The second assertion follows from the definition of function $Z_{i}$.

Remark 1. Since the solutions $x\left(t, \gamma_{*}\right)$ and $x\left(t, \gamma^{*}\right)$ satisfy $x\left(b, \gamma_{*}\right)=x\left(b, \gamma^{*}\right)=$ $B$ it follows that $Z_{i}\left(\gamma_{*}, \gamma^{*}\right)=b$ for some $i \in \mathbb{N}$.

Proposition 2. If $Z_{i}\left(\gamma, \gamma^{*}\right)=b$ for some $i \in \mathbb{N}$ and $\gamma \in\left[\gamma_{*}, \gamma^{*}\right)$ then $x(t, \gamma)$ is a solution of the $B V P(1.1)$, (1.2) and $x(t, \gamma)$ has $i-1$ points of intersection with $x\left(t, \gamma^{*}\right)$ in the interval $(a, b)$.

This assertion is symmetric to the previous one.

Proposition 3. If there exists a solution $x(t, \gamma)$ of the BVP (1.1), (1.2) with $\gamma \in\left(\gamma_{*}, \gamma^{*}\right)$ then $Z_{i}\left(\gamma, \gamma^{*}\right)=b$ and $Z_{j}\left(\gamma, \gamma_{*}\right)=b$ for some $i, j \in \mathbb{N}$.

Proof. Since $x(b, \gamma)=B=x\left(b, \gamma_{*}\right)=x\left(b, \gamma^{*}\right)$, the graphs of all three solutions intersect at $(b, B)$. The assertion follows from definition of $Z_{i}$.

Corollary 1. If $Z_{i}\left(\gamma_{1}, \gamma_{2}\right)=b$ for some $i \in \mathbb{N}$ and some point $\left(\gamma_{1}, \gamma_{2}\right)$ in the interior of the triangle region then both solutions $x\left(t, \gamma_{1}\right)$ and $x\left(t, \gamma_{2}\right)$ either solve the problem or do not solve the BVP.

Proof. Follows from definition of $Z_{i}\left(\gamma_{1}, \gamma_{2}\right)$.

Proposition 4. Suppose solutions $x\left(t, \gamma^{*}\right)$ and $x\left(t, \gamma_{*}\right)$ are of types $n$ and $m$ respectively and $|n-m| \geq 2$. Then there exist at least $|n-m-1|$ more solutions of the $B V P$.

This assertion was proved in [2].

Remark 2. There are examples of equations such that there is a solution of type 1 and a solution of type 0 but there are not more solutions of the problem. The problem $x^{\prime \prime}=\varphi(x), x(0)=x(1)=0$, where

$$
\varphi(x)= \begin{cases}-x^{3}, & x>0 \\ 0, & x \leq 0\end{cases}
$$

has only the trivial solution (of zero type) and a solution without zeros in $(0,1)$ (of type 1).

Remark 3. There are examples of problems that have a continuum of solutions between two given solutions. Consider the problem $x^{\prime \prime}=\psi(x), x(0)=x(\pi)=$ 0 , where

$$
\psi(x)= \begin{cases}1, & x>1 \\ -x, & -1 \leq x \leq 1, \\ -1, & x<-1\end{cases}
$$

This problem has two solutions $u=-\sin t$ and $v=\sin t$ and a continuum of solutions $x(t)=\alpha \sin t$, where $\alpha \in(-1,1)$. There are no solutions of the problem with $x^{\prime}(0)>1$ or $x^{\prime}(0)<-1$. 
It was proved in the paper by L. Erbe that if for any solution $x(t, \gamma)$ of equation (1.1) the respective equation of variations (1.3) is disconjugate then there is no more than a unique solution of the BVP.

Corollary 2. In the set $S(u, v)$ there is a solution with the equation of variations (1.3) such that it is not disconjugate in the interval $(a, b)$.

We believe that the below statement is valid and formulate it as a hypothesis.

Proposition 5. Suppose that for all solutions $x(t, \gamma) \in S(u, v)$ the respective equations of variations (1.3) have the property: any $y(t)$ is such that there are $i$ zeros in the interval $(a, b)$ and $y(b) \neq 0$. Then there are no solutions of $B V P$ in the set $S(u, v)$ except $u$ and $v$.

Remark 4. There are examples of problems that have solutions of the same type. Consider the problem $x^{\prime \prime}=-12 x+x^{3}, x(0)=x(\pi)=0$. It has solutions for $x^{\prime}(0) \simeq \pm 6.3$. These solutions are of type 2 as shows the graph of the respective equations of variations depicted in Figure 4.

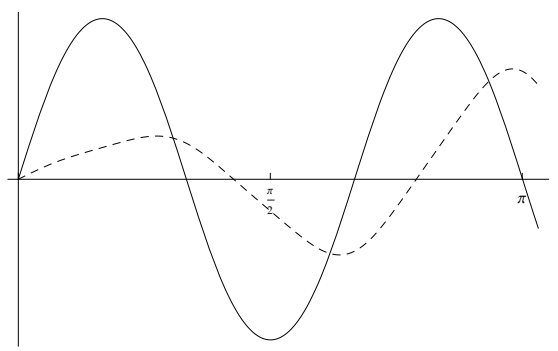

Figure 4. The solution of the problem $x^{\prime \prime}=-12 x+x^{3}, x(0)=x(\pi)=0$ together with the solution $y(t)$ (dashed) of the respective equation of variations.

\section{Interrelations of two solutions of BVP}

We claim that interrelation of two solutions of BVP is not arbitrary.

Proposition 4 says that if types of $u$ and $v$ are different (respectively $n$ and $m$ with $|n-m| \geq 2$ ) then there exist additional solutions. Remark 2 provides the example of no more solutions of BVP if $|n-m|=1$.

The case of two solutions $u$ and $v$ with equal indices is specific. Such solutions cannot intersect arbitrarily. We believe that the number of intersections of graphs of both solutions in that case is approximately the same as their common type.

At the moment we are able to prove the following assertion.

Proposition 6. Let a set $S(u, v)$ be given as above. Suppose both solutions $u$ and $v$ are of type $i$. Let also the following hold: either $Z_{i}\left(\gamma_{*}, \beta\right)<b<$ $Z_{i+1}\left(\gamma_{*}, \beta\right) \forall \beta \in\left(\gamma_{*}, \gamma^{*}\right)$ or $Z_{i}\left(\alpha, \gamma^{*}\right)<b<Z_{i+1}\left(\alpha, \gamma^{*}\right) \forall \alpha \in\left(\gamma_{*}, \gamma^{*}\right)$. 
Then the difference $u(t)-v(t)$ has at least $i-1$ and at most $i$ zeros in the interval $(a, b)$.

Proof. Consider the first case $Z_{i}\left(\gamma_{*}, \beta\right)<b<Z_{i+1}\left(\gamma_{*}, \beta\right) \forall \beta \in\left(\gamma_{*}, \gamma^{*}\right)$. This means that the difference $u(t)-x(t, \gamma)$ has exactly $i$ zeros in the interval $(a, b)$ and $u(b)-x(b, \gamma) \neq 0$ for any $\gamma \in\left(\gamma_{*}, \gamma^{*}\right)$. Recall the triangle region depicted in Fig. 1. Changing $\gamma$ from $\gamma_{*}$ to $\gamma^{*}$ means going from vertex $\mathrm{V}$ to $\mathrm{W}$. Therefore at $\mathrm{W}$ one has either $Z_{i}\left(\gamma_{*}, \gamma^{*}\right) \leq b<Z_{i+1}\left(\gamma_{*}, \beta\right)$ or $Z_{i}\left(\gamma_{*}, \gamma^{*}\right)<b \leq Z_{i+1}\left(\gamma_{*}, \beta\right)$. This means that either the difference $u(t)-v(t)$ has at least $i-1$ and at most $i$ zeros in the interval $(a, b)$.

Remark 5. It was proved in [7] that quasilinear problem of the form

$$
\left(l_{2} x\right)(t)=\varphi\left(t, x, x^{\prime}\right), \quad x(a)=A, x(b)=B
$$

always has solutions of the type which corresponds to the oscillatory type of the linear part $\left(l_{2} x\right)(t):=x^{\prime \prime}+p(t) x^{\prime}+q(t) x$. If there are multiple (more than one) solutions of the problem (4.1) then $x_{\max }(t)$ and $x_{\min }(t)$ are of this type. Recall that $x_{\max }(t)$ (resp.: $x_{\min }(t)$ ) is a solution of the problem (4.1) that has maximal (resp.: minimal) value of $x^{\prime}(a)$.

Therefore the following corollary of Proposition 6 is valid.

Corollary 3. Suppose $x_{\min }$ and $x_{\max }$ are solutions of a quasilinear problem (4.1) as described above and the type of the linear part is $i$.

Then the difference $x_{\max }(t)-x_{\min }(t)$ has at least $i-1$ and at most $i$ zeros in the interval $(a, b)$.

\section{Conclusions}

In case of multiple solutions of the BVP (1.1), (1.2) it is reasonable to consider types of solutions expressed in terms of oscillatory behaviour of the respective equations of variations.

If these solutions are of different types then there exist additional solutions of the BVP (1.1), (1.2). Namely, if the Dirichlet problem has solutions $x_{n}$ and $x_{m}$ of different types $(n \neq m)$ and $|m-n| \geq 2$, then there exist at least $|m-n|-1$ more others solutions of this BVP.

The relative positions of different solutions of BVP depend on the structure of sets defined by $Z_{i}=b$.

\section{Acknowledgements}

The authors would like to thank the referee for carefully reading the paper.

\section{References}

[1] C. De Coster and P. Habets. Two-point boundary value problems: lower and upper solutions, volume 205 of Mathematics in Science and Engineering. Elsevier, Amsterdam, Netherlands, 2006. 
[2] M. Dobkevich and F. Sadyrbaev. Types and multiplicity of solutions to SturmLiouville boundary value problem. Mathematical Modelling and Analysis, 20(1):18, 2015. http://dx.doi.org/10.3846/13926292.2015.996259.

[3] L.H. Erbe. Nonlinear boundary value problems for second order differential equations. Journal of Differential Equations, $\mathbf{7}(3): 459-472,1970$. http://dx.doi.org/10.1016/0022-0396(70)90094-X.

[4] H.-W. Knobloch. Comparison theorems for nonlinear second order differential equations. Journal of Differential Equations, 1(1):1-26, 1965. http://dx.doi.org/10.1016/0022-0396(65)90025-2.

[5] M. Krasnoselskii, A. Perov, A. Povolockii and P. Zabreiko. Plane vector fields. Fizmatgiz, Moscow, 1966. (in Russian)

[6] F.G. Tricomi. Differential Equations. Blackie \& Son Ltd, London, 1962. (in Russian)

[7] I. Yermachenko and F. Sadyrbaev. Quasilinearization and multiple solutions of the Emden-Fowler type equation. Mathematical Modelling and Analysis, 10(1):41-50, 2005. http://dx.doi.org/10.1080/13926292.2005.9637269. 